Revue d'histoire de l'Amérique française

Q REVUE D.HISTOIRE DE L'AMÉRIQUE FRANÇAISE

\title{
MARSHALL, Bill, Quebec National Cinema (Montreal, McGill-Queen's University Press, 2001), 371 p.
}

\section{Catherine Saouter}

Volume 56, numéro 1, été 2002

URI : https://id.erudit.org/iderudit/007229ar

DOI : https://doi.org/10.7202/007229ar

Aller au sommaire du numéro

Éditeur(s)

Institut d'histoire de l'Amérique française

\section{ISSN}

0035-2357 (imprimé)

1492-1383 (numérique)

Découvrir la revue

Citer ce compte rendu

Saouter, C. (2002). Compte rendu de [MARSHALL, Bill, Quebec National Cinema (Montreal, McGill-Queen's University Press, 2001), 371 p.] Revue d'histoire de

l'Amérique française, 56(1), 98-100. https://doi.org/10.7202/007229ar d'utilisation que vous pouvez consulter en ligne.

https://apropos.erudit.org/fr/usagers/politique-dutilisation/ 
Par ailleurs, une mise en contexte des modalités de l'alliance francoiroquoise aurait favorisé la compréhension des motivations amérindiennes. Quel est, par exemple, le rôle des présents dans cette alliance? Compte tenu de leur emplacement géographique, au cœur de la colonie française, quelle était la marge de manœuvre réelle des Iroquois?

Sur le plan méthodologique, quelques lacunes sont à souligner: l'approche strictement événementielle de l'auteur apporte une quantité de détails sur le déroulement des batailles, mais nuit à l'interprétation d'ensemble. De plus, dans le but de pallier le déséquilibre des noms européens et amérindiens dans les sources, l'auteur adopte une méthode fort curieuse : il gomme volontairement le nom de protagonistes européens d'importance "mineure», tout en avouant que cela ne règle pas le problème de l'anonymat des Autochtones. Pourquoi alors éliminer des détails pertinents?

En somme, l'ouvrage de MacLeod contient une analyse détaillée de la participation des Iroquois de la vallée du Saint-Laurent aux batailles de la guerre de Sept Ans, mais ne remplit que partiellement son objectif de refaire l'histoire de la guerre du point de vue des Iroquois.

MAXIME GOHIER

Département d'histoire Université du Québec à Montréal

MARSHALL, Bill, Quebec National Cinema (Montreal, McGill-Queen's University Press, 2001), $371 \mathrm{p}$.

D'entrée de jeu, il faut signaler à quel point l'ouvrage Quebec National Cinema se démarque des littératures auxquelles nous ont habitués trop de critiques et de chercheurs du cinéma en général ou du cinéma québécois en particulier. Il ne s'agit pas ici de l'apologie esthétisante ou dogmatique d'un cinéma national, mais bien plutôt d'une magistrale étude de l'idée de nation telle qu'elle est vécue et symbolisée par un des groupes de cette planète, à travers l'exploration minutieuse d'un lieu culturel particulièrement propice à en fournir des représentations, à savoir le cinéma de fiction.

Bill Marshall, professeur à l'Université de Glasgow, par la passion qu'il manifeste pour la culture et la nation québécoises, rappelle parfois ces accents qui, en d'autres temps - et bien sûr sous d'autres espèces furent ceux de Mason Wade, et on se félicitera de l'existence de ces 
Zadig dont l'enthousiasme et l'extériorité permettent de recevoir ces regards clairvoyants, autant bienveillants que solidement critiques.

La lecture du premier chapitre, Producing and Envisioning the Nation, est essentielle à la bonne compréhension de la problématique posée par l'auteur, des cadres de référence sur lesquels il s'appuie et de la thèse qu'il va développer. En effet, cette étude n'a pas tant pour objet le cinéma québécois, que ce que Bill Marshall appelle the imagined community, c'est-à-dire l'ensemble des représentations effectuées par la nation, preuve intrinsèque de l'élaboration du sentiment de nation. Reprenant, pour la définition de cet objet, les travaux de Benedict Anderson, Bill Marshall décrit quatre éléments clés qui président à cette élaboration: la reconnaissance, l'espace, le temps et l'ontologie/identité. Ce sont ces quatre éléments qui conduisent l'étude plutôt que les divisions usuelles. On ne trouvera donc pas d'ordonnancement rigoureusement chronologique, ni de classification suivant les modes de production ni encore par genres ou par auteurs. On y trouvera plutôt une justification fine de la valeur de l'étude de la cinématographie pour décrypter ces représentations et l'intérêt, pour analyser la relation entre l'imagined community et la nation, de se pencher sur le cas du Québec.

Les différents volets de l'étude, dont l'ordre de lecture importe guère, sont encadrés par quatre films proposés comme les bornes d'un mouvement qui va de la Révolution tranquille à l'actuelle mondialisation. Les films Pour la suite $d u$ monde et $\dot{A}$ tout prendre sont posés comme fondateurs, véritables paradigmes modernes de la représentation nationale, autant dans leurs cohérences que dans leurs contradictions. À l'autre extrémité, Le déclin de l'empire américain et Le confessionnal sont les symptômes d'une remise en question postmoderne. Entre ces bornes, la thèse explore tout le répertoire cinématographique québécois, des films les plus confidentiels jusqu'aux meilleurs succès du box-office, en passant par les plus élitistes et les pires navets, offrant des pages remarquables sur Maria Chapdelaine, Les Boys, Elvis Gratton, La sarrazine et autres Bons débarras. Jean-Claude Lauzon ou Louis Saïa, Claude Fournier ou Léa Pool, Gilles Carle ou Paul Tana, aucun réalisateur n'est laissé pour compte: les ouvres de chacun sont toutes significatives parce qu'elles contribuent à ce travail de représentation, d'allégorie d'une nation traversée par ses mythes, ses diktats, ses connivences, ses antagonismes et circonvolutions.

Traitant des relations entre France et Québec, entre sexe et nation, de la représentation de l'autre et du discours de l'autre, autochtone ou 
immigrant, du cinéma de femmes, du cinéma d'auteur et du cinéma populaire, l'auteur démontre une connaissance du corpus qui laisse abasourdi. Non seulement Bill Marshall semble avoir tout vu mais, par ses mises en contexte, laisse aussi constater une connaissance de l'histoire et de l'actualité québécoise minutieusement documentée. Cela l'autorise à livrer une analyse des caractéristiques de la nation québécoise, des discours national et nationaliste, révélant des spécificités et des enjeux dont la complexité incite largement à réinterroger autant le discours dominant, du mythe des origines à l'épiphanie référendaire, que les discours alternatifs, de l'anhistoricité postmoderne à l'indifférence politique.

En toute logique, Quebec National Cinema est en rupture avec la critique et la recherche faite à l'intérieur de la nation, parties prenantes du travail de représentation. Il est aussi en rupture avec le champ des études cinématographiques pour lesquelles le film, plus souvent qu'autrement, est une fin en soi. Fidèle en tout moment du texte à la thèse annoncée, il faut lire l'analyse que l'auteur consacre au phénomène des célébrités qu'il distingue d'un impossible star system en sol québécois, et apprécier le traitement qu'il réserve à des expressions comme notre Dominique Michel nationale, ou à des images comme la bouche ouverte de Roy Dupuis.

Ce serait trahir l'auteur que de réserver cet ouvrage aux seuls cinéphiles. Cependant, il serait peut-être à conseiller de réviser ses classiques avant d'en entreprendre la lecture. Il reste alors à regretter qu'une filmographie dûment compilée n'accompagne la bibliographie et l'index, offrant ainsi un repérage plus aisé des visionnements ou revisionnements à envisager. Une bonne connaissance du corpus est en effet indispensable avant de penser ouvrir un débat contradictoire avec l'auteur.

CATHERINE SAOUTER Département de communications Université du Québec à Montréal

MOOGK, Peter N., La Nouvelle France. The Making of French Canada - A Cultural History (East Lansing, Michigan State University Press, 2000), xx-340 p.

Comme l'indique le sous-titre de ce livre, Peter Moogk estime que plusieurs caractéristiques de la société canadienne-française (comprendre québécoise) du $\mathrm{xx}^{\mathrm{e}}$ siècle s'expliquent par des valeurs datant de l'époque 